\title{
Análise dos determinantes da felicidade dos associados da lar cooperativa agroindustrial
}

\author{
Analysis of the determinants of happiness in the LAR Cooperative \\ Agroindustry
}

\author{
Roberta Vedana $^{1}$ (D), Marcos de Oliveira Garcias ${ }^{2}$ (D), Pery Francisco Assis Shikida ${ }^{1}$ (D), \\ Mary Paula Arends-Kuenning ${ }^{3}$ (D) \\ ${ }^{1}$ Universidade Estadual do Oeste do Paraná (UNIOESTE), Toledo (PR), Brasil. E-mails: robertavedana@hotmail.com; \\ peryshikida@hotmail.com \\ ${ }^{2}$ Universidade Federal da Integração Latino-Americana (UNILA), Foz do Iguaçu, (PR), Brasil. E-mail: \\ marcos.o.garcias@gmail.com \\ 3University of Illinois, Champaign (IL), United States. E-mail: marends@illinois.edu
}

\begin{abstract}
Como citar: Vedana, R., Garcias, M. O., Shikida, P. F. A. \& Arends-Kuenning, M. P. (2021). Análise dos determinantes da felicidade dos associados da lar cooperativa agroindustrial. Revista de Economia e Sociologia Rural, 59(1), e238882. https://doi.org/10.1590/1806-9479.2020.238882
\end{abstract}

Resumo: Sabendo-se que a felicidade é sentida de forma subjetiva e a sua mensuração envolve a avaliação que cada pessoa faz de sua vida, este trabalho objetivou identificar e analisar quais são os determinantes socioeconômicos que influenciam na felicidade dos associados da Lar Cooperativa Agroindustrial. Para tanto, elaboraram-se questões que buscaram captar a percepção individual de felicidade de uma amostra de 150 casais (300 indivíduos) associados à Lar Cooperativa Agroindustrial, distribuídos em 5 municípios da região oeste paranaense em que a Cooperativa atua. Essa análise foi feita em duas etapas. Na primeira etapa, qualitativa, identificaram-se palavras que, na percepção do indivíduo, descrevem a felicidade. Na segunda etapa, quantitativa, fez-se uso de variáveis que descrevessem os processos citados pelos indivíduos mensurados com o uso de um modelo lógite. Observou-se que a idade, sexo, número de horas trabalhadas e a presença de sucessores na atividade agropecuária afetam a probabilidade dos indivíduos se autodeclararem felizes.

Palavras-chave: felicidade, cooperativismo, sucessão familiar.

\begin{abstract}
Happiness is felt in a subjective way and its measurement involves the evaluation that each person makes of his life. In this sense, this study investigates the economic and social determinants that explain the happiness of the members of Lar Cooperativa Agroindustrial. In order to obtain the results, a questionnaire was elaborated that sought to capture the individual perception of happiness in a sample of 150 couples (300 individuals) distributed in 5 municipalities in the western region of Paraná, where the Cooperative operates. Based on these data, this analysis was carried out in two stages. In the first step, qualitative, words were identified that in the individual's perception describe happiness. In the second stage, quantitative, variables that describe the processes cited by the individuals were used in a logit model. The results showed that age, sex, number of hours worked and the presence of successors in farming activities affect the likelihood of individuals declaring themselves happy.
\end{abstract}

Keywords: happiness, cooperativism, family succession.

\section{INTRODUÇÃO E FORMULAÇÃO DO PROBLEMA DE PESQUISA}

O bem-estar é uma das principais fontes motivacionais para a grande maioria das pessoas. Sentir-se bem reflete diretamente no sentimento de felicidade, variável que, cada vez mais, tem se mostrado indispensável para o desenvolvimento socioeconômico dos países, tanto nas suas áreas urbanas quanto rurais.

Para mensurar o progresso de uma sociedade, sendo esta avaliada pelas suas conquistas nos âmbitos econômicos e sociais, existem diversos indicadores. Entre os mais conhecidos 
destacam-se o Produto Interno Bruto (PIB), disseminado mundialmente a partir da década de 1940, além do PIB per capita, Produto Nacional Bruto (PNB) e o Produto Nacional Líquido (PNL) (Sales et al., 2013).

Por abarcar apenas questões quantitativas de natureza puramente econômica, a busca por alternativas capazes de capturar os fatores relacionados ao bem-estar fez surgir, na década de 1970, o conceito de Gross National Happiness (GNH) - Felicidade Interna Bruta (FIB) - o qual foi criado no Butão. A construção desse indicador contou com a contribuição do Programa das Nações Unidas para o Desenvolvimento (PNUD) e abarca nove dimensões, a saber: bem-estar psicológico, uso racional do tempo, vitalidade da comunidade, cultura, saúde, educação, diversidade do meio ambiente, padrão de vida e governança (Del Bianco et al., 2016).

Mais tarde, na década de 1990, foi criado o Índice de Desenvolvimento Humano (IDH), sendo medido em três dimensões básicas: renda, educação e saúde.

Entre outros indicadores sociais, citam-se ainda o Índice de Liberdade Humana (ILH), o Índice de Liberdade Política (ILP) e o Índice de Pobreza Humana (IPH) (Sales et al., 2013).

$\mathrm{Na}$ busca pelo melhor meio de se medir o progresso de uma sociedade, indo além dos indicadores tradicionais e examinando a vida cotidiana das pessoas sob diversas áreas, como saúde, escolaridade, renda, realização pessoal e condições sociais, a Organização de Cooperação e de Desenvolvimento Econômico (Organização de Cooperação e de Desenvolvimento Econômico, 2011) lançou, em 2010, a Better Life Iniciative (Iniciativa para uma Vida Melhor).

Esta iniciativa visa fornecer evidências abrangentes sobre padrões e tendências de bemestar nos países que fazem parte da OCDE e em países subdesenvolvidos. Esta mensuração do bem-estar é feita por meio do Better Life Index (Índice para uma Vida Melhor), índice que é publicado, desde 2011, no relatório How's Life? (Como Vai a Vida?). As 11 dimensões consideradas no cálculo do índice são: comunidade, educação, meio ambiente, engajamento cívico, saúde, moradia, renda, emprego, satisfação pessoal, segurança e equilíbrio entre vida e trabalho (Organização de Cooperação e de Desenvolvimento Econômico, 2011).

Essas variáveis permitem compreender os fatores que promovem o bem-estar das pessoas e avaliar aquilo que ainda precisa ser feito para que os indivíduos alcancem melhores condições de vida. Neste sentido, é interessante notar que o Better Life Index não produz um ranking com os resultados dos países. O intuito desse índice é permitir uma visualização dinâmica do desempenho de cada um deles, de modo que cada variável pode ser facilmente avaliada individualmente e, ao mesmo tempo, comparada entre as nações (Organização de Cooperação e de Desenvolvimento Econômico, 2020).

Uma outra iniciativa, lançada em 2012 pela Organização das Nações Unidas (ONU), produz, anualmente, o Relatório Mundial da Felicidade para analisar a percepção desse sentimento em mais de 150 países. As informações para a elaboração deste Relatório são coletadas pela pesquisa mundial da Gallup World Poll - uma empresa global de análise e consultoria de dados. No Relatório de 2020, que considera os dados de 2017 a 2019, o Brasil ocupa a $32^{a}$ posição, assim como em 2019. Seis variáveis são avaliadas, dentre as quais estão: o PIB per capita, a expectativa de vida, o suporte social, a liberdade para fazer escolhas, a generosidade e a percepção da corrupção (Helliwell et al., 2012).

No âmbito acadêmico, pesquisas internacionais e nacionais vêm sendo realizadas, nas últimas décadas, com o objetivo de mensurar a felicidade e seus determinantes. A limitação metodológica que se impõe a este tipo de trabalho e que gera controvérsias se dá, principalmente, pela dificuldade de comparação do nível de felicidade entre os indivíduos, visto que os dados são obtidos por meio de questionários em que as respostas consistem em autorrelatos e que, portanto, permitem que cada pessoa faça uso de sua própria definição de felicidade. Entretanto, o que se observa é que, apesar das diferenças socioculturais e econômicas, a essência das respostas é a mesma para a maioria das pessoas. Independente da região, as preocupações consistem, em geral, na vida familiar, na saúde, no emprego e na situação financeira (Giannetti, 2002; Corbi \& Menezes-Filho, 2006).

A fim de contribuir com a discussão da temática, este trabalho procurou, por meio de um estudo de caso com a aplicação de questionário, identificar e analisar quais são os 
determinantes socioeconômicos que influenciam na felicidade dos associados da Lar Cooperativa Agroindustrial.

Fundada em 1964, com sede localizada no oeste do estado do Paraná, a Lar é uma das instituições que contribuem para o crescimento desta região, visto que em 2018 faturou $R \$$ 6,4 bilhões, empregou mais de 13 mil funcionários e contou com um quadro de 10.887 associados. Entre as atribuições da cooperativa, incluem-se a difusão de tecnologia, a assistência técnica, a agregação de valor aos produtos, além da promoção de atividades de capacitação para associados e familiares (Lar Cooperativa Agroindustrial, 2020).

Assim como os homens, as mulheres participam ativamente da Cooperativa. Os cursos e palestras com direcionamento ao público feminino, realizados com frequência, são organizados por meio do Programa de Desenvolvimento da Liderança Feminina. Em 2018, a Lar ofereceu, ao longo do ano, 12 treinamentos diferentes abordando assuntos como o cooperativismo, o planejamento e a gestão da propriedade rural, buscando, ainda, enfatizar o protagonismo feminino na liderança cooperativista e familiar. Em 2019, foram realizados 10 treinamentos priorizando os seguintes temas: cooperativismo, liderança, trabalho em equipe, comprometimento, envolvimento, comunicação, motivação e inovações tecnológicas.

A atuação da Lar ao compreender o papel das mulheres e envolvê-las no âmbito cooperativo contribui para a integração não só produtiva e econômica, mas também social do agregado familiar na sociedade.

Tendo em vista o perfil diferenciado de grande parte da região oeste paranaense, especialmente o meio rural, que conta com o acesso a recursos financeiros e produtivos e apresenta elevado nível de capital social e humano, a análise do bem-estar subjetivo torna-se imprescindível para a identificação dos aspectos que, direta ou indiretamente, influenciam no desenvolvimento rural.

Este artigo contém cinco seções, incluindo esta introdução/formulação do problema de pesquisa. Na seção 2 apresenta-se uma revisão da literatura mais recente sobre o tema felicidade. Em seguida, na seção 3, expõem-se os procedimentos metodológicos adotados. A seção 4 contempla a análise resultante da estatística descritiva e do modelo lógite. As conclusões acerca do tema, apresentadas na seção 5, sumarizam este trabalho.

\section{MENSURANDO O BEM-ESTAR SUBJETIVO}

Nos últimos anos, o interesse em entender a essência e os determinantes do bem-estar das pessoas tem sido provocado, principalmente, pela crescente disponibilidade de dados obtidos por meio de pesquisas baseadas na percepção do indivíduo sobre a sua satisfação com a vida. Análises com dados subjetivos podem fornecer uma avaliação pessoal da saúde, escolaridade, renda, realização pessoal e condições sociais de uma pessoa. Esse tipo de investigação é especialmente importante para traçar o curso de políticas públicas, sendo estas planejadas de maneira a atender as demandas específicas dos meios urbano e rural e a melhorar o bem-estar dos indivíduos.

Neste sentido, a compreensão dos determinantes que marcam as diferenças urbanorurais na satisfação de vida das pessoas entre os países foi estudada por Easterlin et al. (2011). Os resultados sugeriram que, em países menos desenvolvidos, existem lacunas substanciais em renda, educação e emprego, as quais favorecem a satisfação de vida nas áreas urbanas em detrimento da vida no meio rural, apesar dos problemas urbanos como a poluição e o congestionamento, por exemplo. Em níveis de desenvolvimento mais avançados, esses diferenciais econômicos tendem a desaparecer e as áreas rurais se aproximam ou até excedem a satisfação da vida urbana.

Por meio de extensa revisão da literatura existente sobre o bem-estar subjetivo, Boarini et al. (2012) mostraram que a renda, a saúde, o emprego, as relações sociais, a habitação, a educação e a atuação do governo têm forte correlação positiva sobre a satisfação dos indivíduos com a vida. Adicionalmente, a partir dos dados da pesquisa mundial da Gallup, encontraram resultados significativos da legislação de proteção ao emprego, do seguro desemprego e das políticas de saúde sobre o bem-estar subjetivo dos indivíduos nos países da OCDE. 
Em estudo realizado no Butão, país onde o índice FIB foi criado, Santos (2013) analisou a redução da pobreza nos anos de 2003 e 2007. Os dados utilizados foram provenientes da pesquisa Bhutan Living Standard Survey (BLSS), realizada pelo National Statistics Bureau (NSB), seguindo os critérios da metodologia desenvolvida pelo Banco Mundial. Estes critérios estão relacionados às condições básicas como saúde, educação, acesso à eletricidade, água potável, saneamento melhorado, espaço suficiente por pessoa na residência, acesso a estradas e propriedade da terra. A maioria desses indicadores, curiosamente, como salienta a autora, foi também identificada como fonte de felicidade pela mensuração do FIB em 2007.

Os resultados obtidos para o país, localizado no sul da Ásia, essencialmente rural e em processo de desenvolvimento, indicaram que, durante o período de análise, houve uma redução significativa da pobreza. Essa redução se deu, principalmente, pela queda da intensidade da pobreza entre as pessoas que eram menos intensamente pobres e, em menor grau, entre aquelas que eram mais intensamente pobres. Em termos gerais, sugere-se que a redução da pobreza e da privação de condições básicas de vida estão atreladas à elevação da felicidade da população butanesa.

Também para o Butão, Samdup et al. (2019) procuraram refinar o conceito do FIB para propriedades rurais. Os dados técnicos, sociais, econômicos e ambientais foram coletados por meio de questionários em 183 domicílios nos anos de 2000, 2004 e 2015. Os autores constataram que, entre 2004 e 2015, os índices sociais apresentaram queda e destacam que os principais desafios políticos para a implementação do conceito do FIB nas áreas rurais são o aumento da migração do meio rural para o urbano, ocasionando a escassez de mão de obra agrícola e a necessidade de um desenvolvimento socioeconômico mais equitativo.

$\mathrm{Na}$ China, o desenvolvimento econômico das cidades atraiu muitos trabalhadores do campo. Esses trabalhadores, geralmente casados, deixam suas casas e esposas para trabalhar nas cidades e, com isso, as esposas liushou assumem a agricultura e os deveres domésticos. O termo mulheres liushou, o qual, ao se realizar uma tradução livre do chinês, significa "fique para trás", foi criado para designar esse grupo. A partir dessa constatação, Liang et al. (2013) se interessaram em investigar os fatores que afetam a felicidade de mulheres liushou em partes ocidentais da China. Os dados para a pesquisa foram coletados por meio de entrevistas com mulheres liushou, entre 18 e 60 anos de idade, no distrito de Yangling, na província de Shaanxi, no período de abril a novembro de 2011. Com base na amostra investigada, constatou-se que a situação financeira, a personalidade, a eficiência governamental, o relacionamento conjugal e com os sogros são fatores significativos que influenciam a felicidade das mulheres liushou. Essa descoberta demonstra que as mulheres chinesas estão dispostas a sacrificar o seu interesse pessoal pelo de suas famílias (Liang et al., 2013).

A busca pelos fatores associados com o nível de felicidade das famílias produtoras de arroz na região de Kedaf, na Malásia, país do sudeste asiático, demonstrou que a atuação de instituições de assistência técnica consegue influenciar, positiva e significativamente, a felicidade dos agricultores pesquisados, assim como a gestão do tempo de lazer e a propriedade de ativos financeiros e humanos. Influenciam negativamente na felicidade destes produtores os fatores ambientais, como poluição, degradação da terra e disposição de resíduos. Os resultados também sugerem que o fator de manejo agrícola, indicado por variáveis como o uso efetivo de máquinas, controle de pragas e ervas daninhas, não é capaz de aumentar a felicidade dos produtores de arroz, visto que grande parte deles são pequenos agricultores e não possuem capital financeiro suficiente para incorporar a tecnologia no cultivo do arroz (Kamaruddin et al., 2013).

Segundo Markussen et al. (2017), muitos países em desenvolvimento estão passando por um processo de transformação no qual parte dos trabalhadores estão migrando da agricultura para outros setores. Neste sentido, os autores procuraram investigar as consequências desse processo para o bem-estar subjetivo de trabalhadores agrícolas (que atuam na propriedade da família), de assalariados e de autônomos (que atuam em atividades não agrícolas).

Utilizando dados para a população rural do Vietnã, Markussen et al. (2017) verificaram que o trabalho agrícola tem efeito significativamente positivo para o bem-estar subjetivo, enquanto o trabalho assalariado e o autônomo têm efeitos negativos. Esses resultados 
demonstram consistência com a discussão teórica apresentada pelos autores ao afirmar que trabalhar na propriedade familiar pode aumentar os sentimentos de competência e autonomia, ao passo que a proximidade e o relacionamento com a família contribuem para melhorar a sensação de bem-estar e felicidade.

O processo de envelhecimento populacional existente em diferentes partes do mundo, especialmente nas áreas rurais, levou Lobos et al. (2015) a explorarem as diferenças nos determinantes do bem-estar e de felicidade entre homens e mulheres idosos, na faixa etária de 60 a 90 anos de idade, que vivem em áreas rurais na região do Maule, no Chile. Os resultados mostram que a satisfação relacionada à alimentação, percepção de saúde e funcionalidade está significativamente ligada à felicidade individual em ambos os grupos de gênero. Um coeficiente positivo para a idade e um coeficiente negativo para o quadrado da idade sugerem uma relação invertida em $U$ entre idade e felicidade no grupo feminino, com um ponto de inflexão na idade de 77,5 anos.

O bem-estar da população residente nas áreas rurais é reconhecido como um dos principais objetivos das políticas de desenvolvimento sustentável na Europa. Tendo isto em vista, Surová et al. (2016) realizaram um estudo em dois municípios do sul de Portugal para verificar a relação entre as características agrícolas locais e os níveis de bem-estar subjetivo relatados pelos residentes rurais entrevistados. Os resultados mostraram que a satisfação com a vida e o bem-estar subjetivo estão, positiva e significativamente, correlacionados à qualidade dos alimentos produzidos localmente, práticas agrícolas, paisagem e ao meio ambiente.

Os determinantes empíricos da felicidade para cinco países, com uma análise mais aprofundada para o Brasil, foram estimados por Corbi \& Menezes-Filho (2006). Os resultados sugerem correlação positiva e significativa entre felicidade e renda. Na maioria dos casos, a felicidade esteve positivamente correlacionada com o casamento. Além disso, a felicidade apresentou uma relação convexa entre idade e felicidade, com ponto de mínimo por volta dos 54 anos.

Lima (2007), em estudo especificamente para o Brasil, confirmou esses resultados, ressaltando que o desemprego contribui negativamente à felicidade individual e, em comparação com os homens, as mulheres se mostraram menos felizes.

Já os determinantes econômicos e sociais que explicam os níveis de felicidade no município de Campo Grande (MS), testados por meio de um modelo probit, segundo Aydos et al. (2017), são a escolaridade, a idade, o estado civil e o estado de origem do entrevistado. A pesquisa foi realizada com dados obtidos a partir da aplicação de questionários no estilo survey para uma amostra de 600 indivíduos. Identificar estes determinantes, de acordo com os autores, pode auxiliar na elaboração de políticas públicas, possibilitando maior retorno aos investimentos realizados.

Em análise para o município de Cascavel, Rodrigues \& Shikida (2005) avaliaram a relação da felicidade com o dinheiro, por meio de aplicação de questionários. Os resultados demonstraram que os três principais aspectos caracterizadores da felicidade também foram dispersos. A saúde foi a principal palavra citada pelos entrevistados, sendo seguida por: família; realização profissional/emprego; dinheiro/renda/bens; realização no amor; paz/tranquilidade; outros valores pessoais e amizade/relacionamento. Ao se agregar a realização profissional/emprego com o dinheiro/renda/bens, compilados no item economia, o item saúde continuou a ser o primeiro, sendo seguido por economia e, em terceiro, o item família. Outra constatação deste estudo está no fato de a relação da felicidade com a economia ser mais dicotômica ao se considerar o nível de renda. Indivíduos possuidores de rendas mais elevadas declararam que o dinheiro não traz felicidade. Por outro lado, indivíduos com rendas mais baixas afirmaram que o dinheiro contribui positivamente para a sua felicidade.

A utilização do FIB, no Brasil se deu pela adaptação desta metodologia aplicada para identificar quais as variáveis que teriam maior influência na felicidade dos trabalhadores das áreas urbana e rural do município de Cascavel-PR. Os entrevistados foram questionados sobre a frequência com que praticavam atividades físicas; se tinham boas noites de sono, sendo este tranquilo e profundo; como era utilizado o tempo no trânsito, no trabalho, nas atividades educacionais, no lazer etc. Entre os aspectos que afetaram positivamente a 
felicidade dos indivíduos, têm-se: ser otimista, praticar exercícios físicos e participar de algum grupo social. Com relação à questão sobre o que deixava os entrevistados mais felizes, observou-se a preferência por fatores não materiais, especialmente os relacionados à família, aos bons sentimentos, boas práticas sociais, saúde, solidariedade e à espiritualidade (Del Bianco et al., 2016).

O que se observa é que, apesar das diferenças econômicas, sociais e culturais, especialmente entre países orientais e ocidentais, a essência das respostas é praticamente a mesma para a maioria das pessoas. A vida familiar, a saúde, a renda e o emprego são os fatores que, independente da região, acabam sendo determinantes para o bem-estar e o sentimento de felicidade humana.

\section{PROCEDIMENTO METODOLÓGICO}

Nesta seção estão descritos os procedimentos metodológicos adotados na obtenção dos dados para este trabalho. O presente artigo tem uma abordagem qualitativa e quantitativa com a realização de um estudo de caso e procedimentos de pesquisa de campo por meio de entrevistas do tipo survey (Gerhardt \& Silveira, 2009).

Foram entrevistados, ao longo dos meses de fevereiro e dezembro de 2019, 150 casais heteronormativos (300 indivíduos) associados à Lar Cooperativa Agroindustrial, os quais se encontram distribuídos em 5 municípios da região oeste paranaense em que a cooperativa atua. Esta amostra é estatisticamente representativa para um nível de 95\% de confiança e margem de erro de $5 \%$.

A análise qualitativa, segundo Gil (2002), preocupa-se em identificar fatores que influenciam na ocorrência de determinados fenômenos. Nesse sentido, este trabalho busca compreender os principais aspectos caracterizadores da felicidade dos associados a uma cooperativa agroindustrial.

Para tanto, os entrevistados foram questionados a respeito das três palavras que, em ordem de importância, caracterizam a sua felicidade. Foi feita também a análise para a resposta dos entrevistados sobre as três palavras, novamente em ordem de importância, que estes indicaram não gostar em suas vidas e que, portanto, não são fontes de felicidade.

A partir da análise descritiva destes aspectos, estimou-se um modelo quantitativo na tentativa de identificar quais variáveis afetariam ou não a probabilidade dos respondentes se considerarem felizes. A estratégia empírica utilizada é a estimação por máxima verossimilhança de um modelo lógite ${ }^{1}$, em que a variável dependente é caracterizada pela condição da pessoa ser feliz ou não. $O$ modelo estimado é apresentado na Equação 1 a seguir:

felicidade $=\alpha+\beta_{1}$ idade $+\beta_{2}$ sexo $+\beta_{3}$ ensino_médio $+\beta_{4}$ ensino_superior $+\beta_{5}$ habilitação +

$\beta_{6}$ renda1 $+\beta_{7}$ renda $2+\beta_{8} n$ filhos $+\beta_{9} n \_$sucessão $+\beta_{10}$ grupo_religioso $+\beta_{11}$ treinamento +

$\beta_{12}$ trabalho_over $+\beta_{13}$ horas_trabalho

As variáveis utilizadas para verificar quais são os determinantes para a felicidade dos indivíduos são apresentadas no Quadro 1.

As variáveis selecionadas para a estimação do modelo lógite, as quais explicam a felicidade dos pesquisados, foram: idade, sexo, nível de instrução, treinamentos, habilitação para dirigir (proxy para liberdade e independência para fazer escolhas), renda, número de filhos e sucessão na agricultura (proxies para família), participação em grupos religiosos, percepção da rotina de trabalho e horas trabalhadas (proxies para trabalho/emprego). Tais variáveis são frequentemente utilizadas pela literatura e testadas como fatores determinantes para a felicidade.

\footnotetext{
${ }^{1}$ Para mais detalhes sobre o modelo lógite, ver Greene (2003).
} 
Quadro 1. Variáveis utilizadas no modelo

\begin{tabular}{|c|c|}
\hline Variável & Descrição \\
\hline felicidade & dummy igual a (1) se o entrevistado se considera feliz e (0) caso contrário. \\
\hline idade & anos da pessoa, contínuo. \\
\hline sexo & dummy igual a (1) se o entrevistado é do sexo masculino e (0) caso contrário. \\
\hline ensino médio & $\begin{array}{l}\text { dummy igual a (1) se o entrevistado possui ensino médio completo ou superior } \\
\text { incompleto e (0) caso contrário. }\end{array}$ \\
\hline ensino superior & $\begin{array}{l}\text { dummy igual a (1) se o entrevistado possui ensino superior completo ou pós- } \\
\text { graduação e (0) caso contrário. }\end{array}$ \\
\hline habilitação & $\begin{array}{l}\text { dummy igual a (1) se o entrevistado possui habilitação para conduzir veículo e } \\
\qquad \begin{array}{l}\text { (0) caso contrário. }\end{array}\end{array}$ \\
\hline rendal & $\begin{array}{c}\text { dummy igual a (1) se a renda familiar é de } 6 \text { a } 9 \text { salários mínimos mensais e (0) } \\
\text { caso contrário. }\end{array}$ \\
\hline renda 2 & $\begin{array}{l}\text { dummy igual a (1) se a renda familiar é superior a } 9 \text { salários mínimos mensais e } \\
\qquad(0) \text { caso contrário. }\end{array}$ \\
\hline$n_{-}$filhos & número de filhos do entrevistado. \\
\hline$n_{-}$sucessão & $\begin{array}{l}\text { número de filhos que pretendem dar continuidade às atividades agropecuárias } \\
\qquad \text { da família. }\end{array}$ \\
\hline grupo_religioso & $\begin{array}{l}\text { dummy igual a (1) se o entrevistado participa de grupo religioso e (0) caso } \\
\text { contrário. }\end{array}$ \\
\hline treinamento & $\begin{array}{c}\text { dummy igual a (1) se o entrevistado participa de treinamentos oferecidos pela } \\
\text { cooperativa e (0) caso contrário. }\end{array}$ \\
\hline trabalho_over & $\begin{array}{l}\text { dummy igual a (1) se o entrevistado considera ter trabalhado mais que o } \\
\text { habitual no dia anterior e }(0) \text { caso contrário. }\end{array}$ \\
\hline horas_trabalho & número de horas trabalhadas nas últimas 24 horas. \\
\hline
\end{tabular}

Fonte: elaboração dos autores.

\section{RESULTADOS E DISCUSSÕES}

Nesta seção são discutidos os resultados da pesquisa. Primeiramente, ocorre a análise descritiva sobre quais aspectos caracterizam a felicidade dos entrevistados. Na sequência, são apresentados os resultados estimados pelo modelo lógite.

Sobre os aspectos caracterizadores da felicidade, analisados em ordem de importância, em que a $1^{\text {a }}$ opção é mais importante do que a $2^{a}$, e assim sucessivamente, indicaram, de maneira geral, respostas bem dispersas, mostrando uma multiplicidade de combinações de fatores que denotam a felicidade destas pessoas, confirmando a revisão de literatura, como de Rodrigues \& Shikida (2005).

$\mathrm{Na}$ Tabela 1 são apresentados os aspectos caracterizadores da felicidade que mais ocorreram.

Nota-se, a partir da Tabela 1, que família e filhos foram os maiores destaques, tendo sido citados $61,3 \%$ das vezes como primeiro aspecto caracterizador da felicidade e em $34,4 \%$ do total de citações. Na sequência, aparece a realização profissional/emprego com pelo menos uma ocorrência em $12 \%$ das vezes.

Dentre os valores que salientam qualidades humanas, foram citadas: honestidade, fidelidade, gratidão, paciência, humildade etc. Estas ocorreram 11,8\% das vezes. Em seguida, estão as atividades de lazer, que incluem dançar, jogar baralho, ler, passear, pescar, praticar esportes, viajar etc., as quais foram citadas $9,7 \%$ das vezes.

A saúde, frequentemente elencada como uma das principais fontes de felicidade, foi citada na presente pesquisa em $7,8 \%$ das vezes. Com este mesmo percentual $(7,8 \%)$, foram mencionadas amizade e as relações sociais. 
Tabela 1. Ordenação das palavras que caracterizam a felicidade dos entrevistados

\begin{tabular}{|c|c|c|c|c|}
\hline Palavras & $\begin{array}{c}\text { \% vezes em } \\
\text { que } \\
\text { apareceu } \\
\text { como } 1^{\text {a }} \\
\text { opção }\end{array}$ & $\begin{array}{c}\text { \% vezes em que } \\
\text { apareceu como } \\
2^{\mathrm{a}} \text { opção }\end{array}$ & $\begin{array}{l}\text { \% vezes em que } \\
\text { apareceu como } \\
3^{\text {a opção }}\end{array}$ & $\begin{array}{c}\% \text { total de vezes } \\
\text { em que } \\
\text { apareceu }\end{array}$ \\
\hline Família/Filhos & 61,3 & 29 & 13 & 34,4 \\
\hline Realização Profissional/ Emprego & 5 & 17,7 & 13,3 & 12 \\
\hline Valores humanos & 8,3 & 10,3 & 16,7 & 11,8 \\
\hline Lazer & 2,3 & 10,7 & 16 & 9,7 \\
\hline Saúde & 6,7 & 10 & 6,7 & 7,8 \\
\hline Amizade/ Relações sociais & 2 & 11,7 & 9,7 & 7,8 \\
\hline Valores Religiosos & 6,7 & 4,7 & 7 & 6,1 \\
\hline Realização no Amor & 6,7 & 3,6 & 5,3 & 5,2 \\
\hline Dinheiro/Renda/Bens & 1 & 1,3 & 5,7 & 2,7 \\
\hline Preferiu não responder & 0 & 1 & 6,6 & 2,5 \\
\hline
\end{tabular}

Fonte: dados da pesquisa (2020).

Na sequência, aparecem os valores religiosos, que incluem palavras como Deus, fé e religião. Este aspecto foi citado $6,1 \%$ das vezes. A realização no amor, que se refere a satisfação com a vida matrimonial, foi citada como característica para a felicidade em 5,2\% das vezes.

Os aspectos econômicos, analisados em conjunto com o dinheiro, renda e bens, aparecem em $2,7 \%$ das vezes.

Dentre os entrevistados que não sabiam ou preferiram não responder, $1 \%$ não citou uma $2^{a}$ opção de palavra e 6,6\% não mencionaram uma $3^{a}$ opção. Isso quer dizer que todos os entrevistados têm, pelo menos, um aspecto que consideram indispensável para a sua felicidade.

Ao serem questionados sobre o que não gostavam em suas vidas e que, portanto, não contribuem para a sua felicidade, também em ordem de importância, os entrevistados listaram fatores como doenças, adversidades climáticas, política etc., mas se referiram, mais enfaticamente, a aspectos que salientam, principalmente, desvirtudes humanas. A análise dos termos mais frequentes resultou em uma nuvem de palavras ilustrada pela Figura 1.

Os resultados obtidos no presente trabalho foram comparados com literaturas reportadas, como Rodrigues \& Shikida (2005) e Del Bianco et al. (2016) que, além de aplicarem questionários com perguntas semelhantes as deste estudo, realizaram as entrevistas em Cascavel, município do Oeste paranaense, região de abrangência dos municípios visitados para esta pesquisa.

Tendo em vista a proximidade metodológica e regional na qual as pesquisas foram desenvolvidas, observou-se elevada conformidade. Em Rodrigues \& Shikida (2005), o fator Família foi o segundo mais citado, enquanto em Del Bianco et al. (2016) foi o primeiro. Destacam-se ainda os aspectos econômicos e valores pessoais com grande influência no sentimento de felicidade dos entrevistados para estas duas referências. 


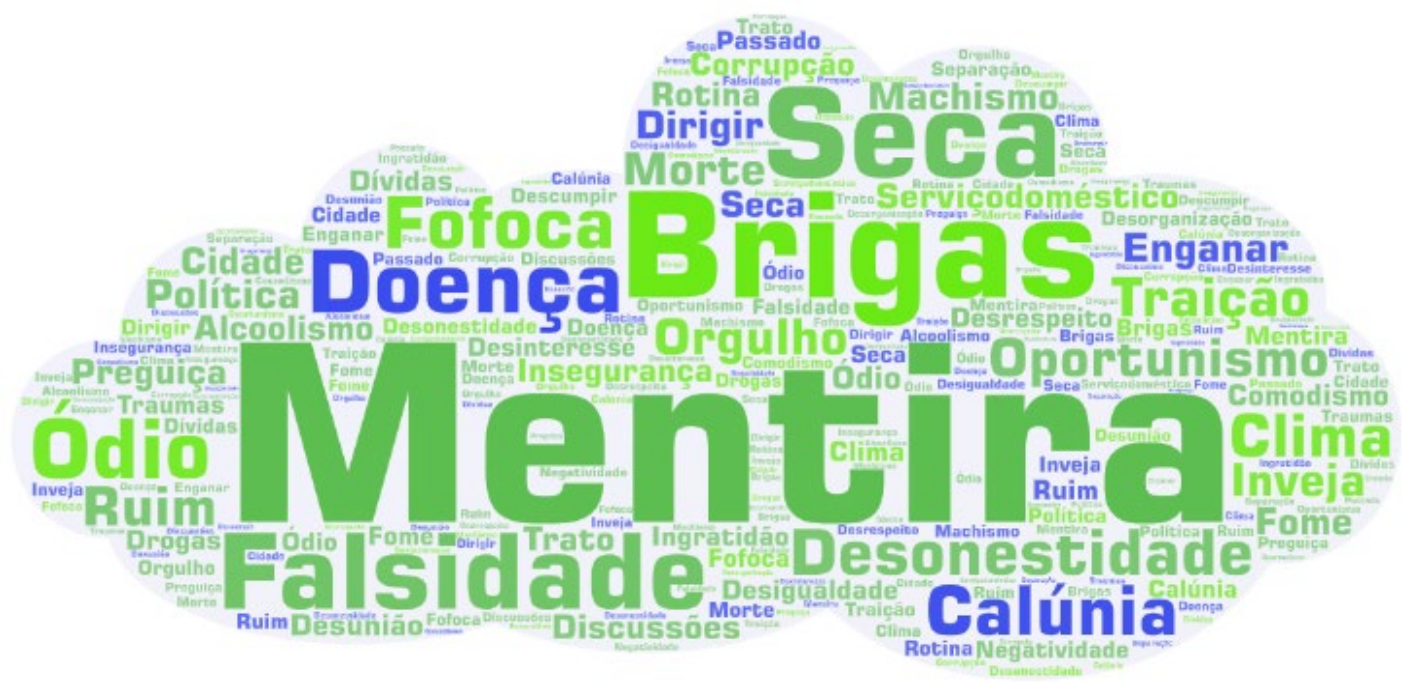

Figura 1. Palavras que não caracterizam a felicidade para os entrevistados. Fonte: dados da pesquisa (2020).

Neste sentido, tendo sido identificados os principais fatores que caracterizam a felicidade, o próximo passo foi analisar estatisticamente se estes aspectos são significativos na determinação deste sentimento dos entrevistados. A Tabela 2 apresenta as estatísticas descritivas das variáveis selecionadas para a estimação do modelo.

Tabela 2. Estatística descritiva das variáveis selecionados para a estimação do modelo lógite

\begin{tabular}{cccccc}
\hline Variáveis & Obs. & Média & Desvio-Padrão & Min & Max \\
Felicidade & 300 & 0,53 & 0,50 & 0 & 1 \\
Idade & 300 & 53,50 & 10,91 & 20 & 85 \\
Sexo & 300 & 0,50 & 0,50 & 0 & 1 \\
Ensino_médio & 300 & 0,29 & 0,45 & 0 & 1 \\
Ensino_superior & 300 & 0,11 & 0,31 & 0 & 1 \\
Habilitação & 300 & 0,85 & 0,36 & 0 & 1 \\
Renda1 (6 a 12) & 300 & 0,24 & 0,43 & 0 & 1 \\
Renda2 (>12) & 300 & 0,10 & 0,30 & 0 & 1 \\
n_filhos & 300 & 2,35 & 0,91 & 0 & 5 \\
n_sucessão & 300 & 1,07 & 0,97 & 0 & 5 \\
Grupo_religioso & 300 & 0,88 & 0,32 & 0 & 1 \\
Treinamento & 300 & 0,81 & 0,39 & 0 & 1 \\
Trabalho_over & 300 & 0,14 & 0,35 & 0 & 1 \\
Horas_trabalho & 300 & 8,57 & 2,85 & 0 & 16 \\
\hline
\end{tabular}

Fonte: dados da pesquisa (2020).

Dos 300 entrevistados, metade são homens e metade são mulheres, pois o questionário foi aplicado nos domicílios de famílias associadas à Lar Cooperativa Agroindustrial. Do total de respondentes, $53 \%$ consideram-se felizes.

Ao se avaliar a escolaridade, $29 \%$ da amostra possui ensino médio completo e apenas $11 \%$ o superior completo. Outra variável incluída como característica pessoal do indivíduo é se ele possui habilitação para dirigir e $85 \%$ da amostra diz possuir a licença. 
Em termos econômicos, 24\% da amostra possui renda mensal entre 6 e 12 salários mínimos e $10 \%$ da amostra possui renda mensal superior a 12 salários. Isso denota o perfil socioeconômico elevado dos entrevistados.

Tabela 3. Coeficientes do modelo lógite estimado pelo método de máxima verossimilhança sobre a felicidade dos entrevistados

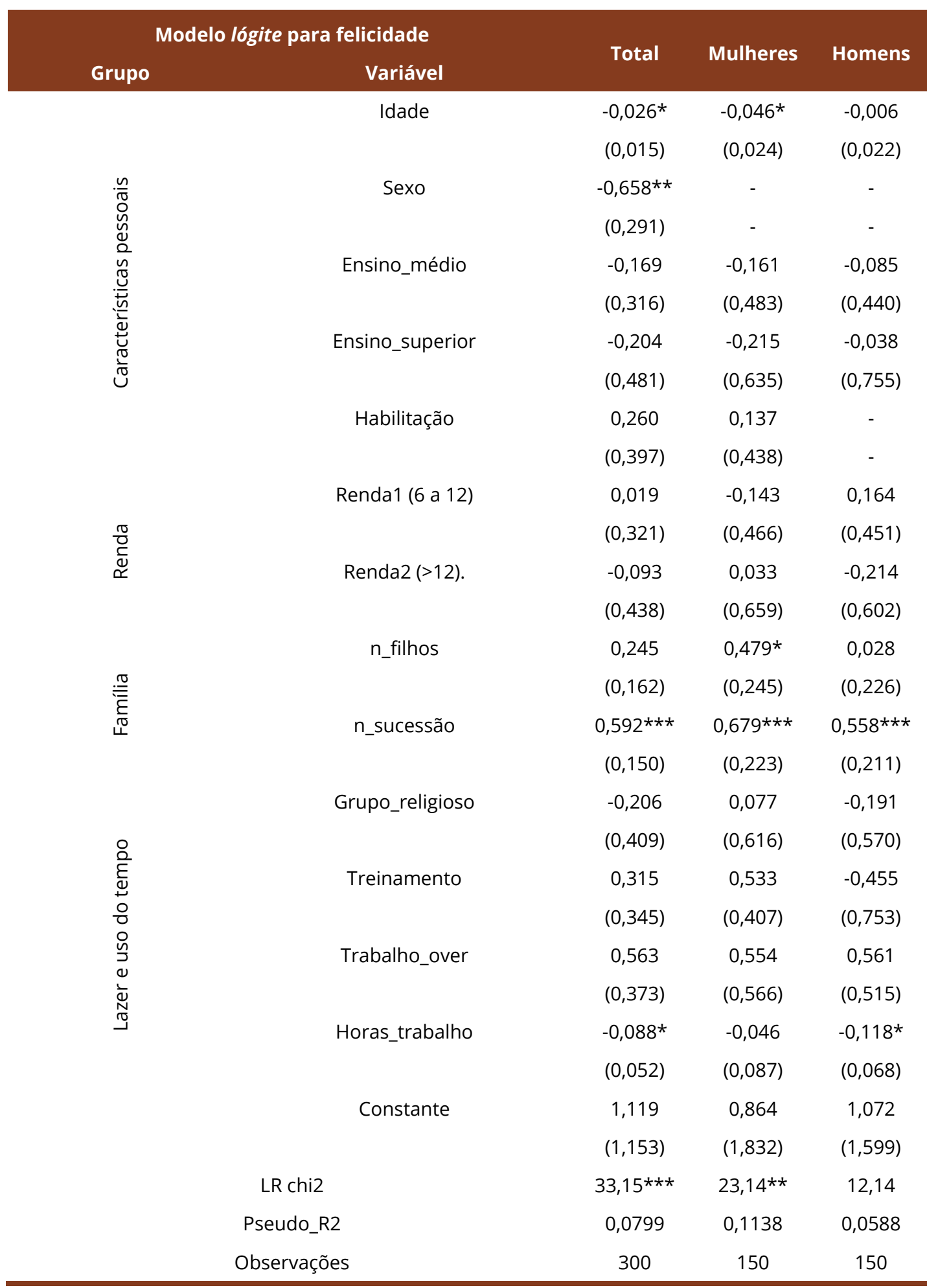

Erro padrão entre parênteses. *** $p<0.01, * * p<0.05, * p<0.1$

Fonte: dados da pesquisa (2020). 
Também foram incluídas perguntas sobre a quantidade de filhos. O número médio de filhos é de 2 a 3 e o maior número encontrado foi famílias com 5 filhos. O número médio de sucessores, por outro lado, é de apenas 1.

Em termos de atividades realizadas pelos entrevistados, de treinamento e lazer, $88 \%$ dizem participar de grupos religiosos e $81 \%$ realizaram algum tipo de treinamento oferecido pela Lar Cooperativa. Em termos de atividades laborais, 14\% dos entrevistados disseram ter trabalhado mais do que o habitual no dia anterior à entrevista e o número médio de horas trabalhadas foi de 8,57 horas.

$\mathrm{Na}$ Tabela 3 são apresentados os resultados dos modelos estimados. Foram realizadas três estimações: a primeira considerando o total de entrevistados (300 observações), a segunda apenas mulheres e a terceira apenas homens. Importante ressaltar que os resultados do modelo estimado apenas para os homens ${ }^{2}$ não foram estatisticamente significativos e, portanto, não serão analisados.

$\mathrm{Na}$ avaliação das variáveis do grupo de características pessoais, idade e sexo foram estatisticamente significativas. O efeito da variável idade foi negativo e evidencia que à medida que a pessoa envelhece, menor a probabilidade de ser feliz. Essa relação também é válida para o modelo estimado apenas com mulheres. Já a variável sexo, no modelo que considera o total de entrevistados, evidencia que homens têm menor probabilidade de se considerarem felizes se comparados às mulheres.

Ao traçar paralelos com os resultados encontrados no presente artigo com a literatura existente, verifica-se que Lobos et al. (2015), em pesquisa sobre o bem-estar de idosos na área rural do Chile, não encontraram diferenças estatisticamente significantes nas pontuações de felicidade, seja por faixa etária ou sexo. Já em Lima (2007), com resultados para o Brasil, observa-se que as mulheres são menos felizes que os homens.

Segundo Helliwell et al. (2012), nos países mais avançados, as mulheres tendem a relatar níveis superiores de felicidade em relação aos homens. Neste sentido, o que pode justificar o resultado encontrado para esta amostra, localizada na região oeste do Paraná, são as condições socioeconômicas diferenciadas, caracterizadas por um modelo de agricultura familiar voltado ao mercado, com um elevado nível de renda, de capital social e humano, com acesso ao crédito, recursos produtivos, assistência técnica especializada e organização cooperativa.

As variáveis de escolaridade e se o indivíduo possui habilitação para dirigir não foram estatisticamente significativas. Esperava-se que a variável habilitação tivesse um efeito positivo e significativo na determinação da felicidade. Acredita-se que a não significância estatística desse parâmetro esteja associada à baixa variabilidade da amostra, já que $85 \%$ dela possui tal licença.

As variáveis de grupo de renda não foram estatisticamente significativas em nenhum dos modelos estimados. Uma possível justificativa para isso é discutida por Helliwell et al. (2012), no Relatório Mundial da Felicidade, em que se argumenta que a renda de uma família contribui para aumentar a satisfação com a vida, mas de forma limitada, visto que outros fatores podem ser mais importantes, como a confiança na comunidade, a saúde mental e física e a qualidade da governança e do estado de direito.

A contribuição da renda como determinante para a felicidade e o bem-estar dos indivíduos foi constatada em grande parte das pesquisas reportadas na seção 2 do presente trabalho, tais como Easterlin et al. (2011), Boarini et al. (2012), Liang et al. (2013), Kamaruddin et al. (2013), em âmbito internacional. Para o Brasil, tem-se Rodrigues \& Shikida (2005), Corbi \& Menezes-Filho (2006), Lima (2007) e Del Bianco et al. (2016). Há um consenso na literatura de que, em geral, pessoas mais ricas tendem a se considerar mais felizes, e os resultados obtidos na presente pesquisa corroboram tal afirmativa.

No grupo de variáveis relacionadas à família, a variável sucessão foi positiva e estatisticamente significativa nos dois modelos avaliados, ou seja, quanto maior o número de filhos que pretendem dar continuidade ao empreendimento agropecuário, maior a

\footnotetext{
${ }^{2}$ O valor do teste chi2 do modelo para homens foi de 12,14, não rejeitando-se a hipótese nula do teste de que pelo menos um dos parâmetros estimados seja diferente de zero. O mesmo teste para os modelos de homens e mulheres e o de apenas para mulheres são estatisticamente significativos.
} 
probabilidade de os pais serem felizes. O alto nível de renda dos entrevistados aliado a propriedades estruturadas e com diversificação de atividade são fatores importantes que podem estar incentivando o processo de sucessão geracional (Kiyota \& Perondi, 2014).

Um outro ponto importante a ser considerado é que a associação à Cooperativa Lar garante o acesso a palestras e treinamentos técnicos que visam discutir o planejamento da sucessão familiar e aproximar a família da gestão da propriedade.

A variável número de filhos foi estatisticamente significativa apenas para o modelo com mulheres, ou seja, para as mães, quanto maior o número de filhos, maior a probabilidade de serem felizes. Este resultado evidencia a importância dos valores familiares citados enfaticamente como um dos aspectos essenciais para uma vida feliz, na análise qualitativa deste estudo.

$\mathrm{Na}$ análise do grupo de variáveis sobre lazer e uso do tempo, apenas a variável número de horas foi estaticamente significativa. Quanto maior o número de horas trabalhadas, menor a probabilidade de os respondentes serem felizes. Relação que vai de encontro com os resultados obtidos por meio dos aspectos caracterizadores da felicidade, dado que a realização profissional, abrangendo palavras que remetem à satisfação com as atividades laborais, ficaram em segundo lugar no total de citações na análise qualitativa.

As variáveis participação em grupos religiosos (grupo_religioso), participação em treinamento oferecidos pela Cooperativa (treinamentos) e percepção da rotina de trabalho (trabalho_over) não foram estatisticamente significativas, embora os valores religiosos e fator trabalho tenham sido citados como importantes fontes de felicidade pelos entrevistados.

\section{CONSIDERAÇÕES FINAIS}

Este trabalho objetivou identificar e analisar quais são os determinantes socioeconômicos que influenciam na felicidade dos associados da Lar Cooperativa Agroindustrial.

Sabendo-se que a felicidade é sentida de forma subjetiva e a sua mensuração envolve a avaliação que cada pessoa faz de sua vida, foram elaboradas questões que buscaram captar a percepção individual de felicidade de uma amostra de 150 casais (300 indivíduos) associados à Lar Cooperativa Agroindustrial, os quais encontram-se distribuídos em 5 municípios da região oeste paranaense em que a cooperativa atua.

De modo geral, pode-se salientar que os aspectos caracterizadores da felicidade mais citados pelos entrevistados foram família e filhos, com maior destaque, seguidos pela realização profissional e pelos valores que salientam qualidades humanas. Na sequência, apareceram as palavras saúde, amizade e relações sociais como importantes fontes de felicidade. Os valores religiosos também apresentaram uma relevante importância para este sentimento. Em seguida, apareceu a realização no amor. Já os aspectos econômicos chamaram a atenção quando analisados em conjunto por meio da agregação entre dinheiro, renda e bens.

A partir desta análise qualitativa, foi possível definir variáveis para serem incluídas em um modelo econométrico, a fim de identificar quais variáveis afetariam ou não a probabilidade dos respondentes se considerarem felizes.

Para isso, foram estimados três modelos: o primeiro considerando o total de entrevistados, o segundo apenas mulheres e o terceiro apenas homens. Entretanto, este último modelo não foi analisado, pois seus resultados não foram estatisticamente significativos.

$\mathrm{Na}$ avaliação das variáveis do grupo de características pessoais, idade e sexo foram estatisticamente significativas. O efeito da variável idade foi negativo e evidencia que à medida que a pessoa envelhece, menor a probabilidade de ela ser feliz. Essa relação também foi validada para o modelo estimado apenas com mulheres. A variável sexo, no modelo para todos os entrevistados, evidencia que os homens têm menor probabilidade de se considerarem felizes se comparados com as mulheres.

No grupo de variáveis relacionadas à família, a variável sucessão foi positiva e estatisticamente significativa nos dois modelos avaliados, ou seja, quanto maior o número de filhos que pretendem dar continuidade ao empreendimento agropecuário, maior a 
probabilidade de os pais serem felizes. Já a variável número de filhos foi estatisticamente significativa apenas para o modelo com mulheres. Neste sentido, para as mães, quanto maior o número de filhos, maior a probabilidade de serem felizes.

$\mathrm{Na}$ análise do grupo de variáveis sobre lazer e uso do tempo, apenas a variável número de horas foi estaticamente significativa. Isso indicou que quanto maior o número de horas trabalhadas, menor a probabilidade de os respondentes serem felizes.

Esses resultados podem auxiliar a cooperativa no desenvolvimento de projetos junto aos associados, uma vez que se conseguiu, nesse estudo, identificar fatores que afetam a probabilidade de se considerarem felizes.

Treinamentos junto aos jovens cooperados e seus pais, que auxiliem no processo de sucessão geracional e atividades voltadas aos membros mais idosos, são exemplos de atividades que a Cooperativa poderá realizar.

\section{Agradecimentos}

Este artigo é fruto de uma cooperação internacional entre a University of Illinois, UNIOESTE, UNILA e Lar Cooperativa Agroindustrial. Os autores são gratos à Lar e aos entrevistados(as) que gentilmente colaboraram com este trabalho.

\section{Referências}

Aydos, L. R., Figueiredo Neto, L. F., \& Teixeira, W. M. (2017). Análise dos determinantes do nível de felicidade subjetiva: uma abordagem local. Interações, 18(1), 137-150.

Boarini, R., Comola, M., Smith, C., Manchin, R., \& Keulenaer, F. (2012). What makes for a better life? The Determinants of subjective well-being in OECD countries - Evidence from the Gallup World Poll (OECD Statistics Working Papers). Paris, France: OECD. Recuperado em Abril de 2020, de http://dx.doi.org/10.1787/5k9b9ltjm937-en

Corbi, R. B., \& Menezes-Filho, N. A. (2006). Os determinantes empíricos da felicidade no Brasil. Revista de Economia Política, 26(4), 518-536.

Del Bianco, T. S., Souza, E. L. C., Oliveira, N. S. M. N., \& Shikida, P. F. A. (2016). A felicidade da população trabalhadora de Cascavel/PR segundo a métrica do índice de Felicidade Interna Bruta. Urbe. Revista Brasileira de Gestão Urbana, 8, 390-406.

Easterlin, R. A., Angelescu, L., \& Zweig, J. S. (2011). The impact of modern economic growth on urbanrural differences in subjective well-being. World Development, 39(12), 2187-2198.

Gerhardt, T. E., \& Silveira, D. T. (Orgs.). (2009). Métodos de pesquisa. Porto Alegre: UFRGS.

Giannetti, E. (2002). Felicidade. São Paulo: Companhia das Letras.

Gil, A. C. (2002). Como elaborar projetos de pesquisa (4a ed.). São Paulo: Atlas.

Greene, W. Econometric analysis (5th ed.). New Jersey: Prentice Hall, 2003.

Helliwell, J., Layard, R., \& Sachs, J. (2012). World happiness report. Columbia: Columbia University. Recuperado em Abril de 2020, de https://www.researchgate.net/publication/233401584

Kamaruddin, R., Ali, J., \& Saad, N. M. (2013). Happiness and its influencing factors among paddy farmers in granary area of Mada. World Applied Sciences Journal, 28, 91-99.

Kiyota, N., \& Perondi, M. A. (2014). Sucessão geracional na agricultura familiar: uma questão de renda? In A. M. Buainain, E. Alves, J. M. Silveira \& Z. Navarro (Eds.), O mundo rural no Brasil do século 21: a formação de um novo padrão agrário e agrícola (pp. 1012-1045). Brasília, DF: Embrapa.

Lar Cooperativa Agroindustrial (2020). Institucional. Recuperado em Abril de 2020, de http://www.lar.ind.br/v4/institucional/index.php

Liang, H., Tang, Y., \& Huo, X. (2013). Liushou women's happiness and its influencing factors in rural China. Social Indicators Research, 117(3), 907-918.

Lima, S. V. (2007). Economia e felicidade: um estudo empírico dos determinantes da felicidade no Brasil (Dissertação de mestrado). Universidade de São Paulo, Ribeirão Preto, SP. 93 f.

Lobos, G., Grunert, K. G., Bustamante, M., \& Schnettler, B. (2015). With health and good food, great life! gender differences and happiness in Chilean rural older adults. Social Indicators Research, 127(2), 865-885. 
Markussen, T., Fibaek, M., Tarp, F., \& Tuan, N. A. (2017). The happy farmer: self-employment and subjective well-being in rural Vietnam. Journal of Happiness Studies, 19, 1613-1636.

Organização de Cooperação e de Desenvolvimento Econômico - OCDE. (2011). OECD launches your better life index. Recuperado em Abril de 2020, de https://www.oecd.org/economy/oecdlaunchesyourbetterlifeindex.htm

Organização de Cooperação e de Desenvolvimento Econômico - OCDE. (2020). OECD Better Life Index. Recuperado em Abril de 2020, de http://www.oecdbetterlifeindex.org/pt.

Rodrigues, O. A., \& Shikida, P. F. A. (2005). Economia e felicidade: elementos teóricos e evidências empíricas. Pesquisa \& Debate, São Paulo, 16(1), 80-120.

Sales, A., Costa, A., Veronese, R., Ferreira, C., \& Resende, L. (2013). Felicidade interna bruta: aplicação e discussão no contexto de cidades de porte médio brasileiras. Revista Cade, 12(1), 59-82.

Samdup, T., Udo, H. M. J., Bokkers, E. A. M., \& Steenstra, F. A. (2019). Linking the Gross National Happiness concept to smallholder farm level: an empirical application. Asian Journal of Agriculture and Development, 16(1), 1-24.

Santos, M. E. (2013). Tracking poverty reduction in Bhutan: income deprivation alongside deprivation in other sources of happiness. Social Indicators Research, 112, 259-290.

Surová, D., Godinho, S., \& Pinto-Correia, T. (2016 july 12-15). Is the local agriculture related to the wellbeing of rural community today? A case from Portugal, Southern Europe. In 12th European IFSA Symposium - 2. Methodology and frameworks of farming systems transformation. Newport, Shropshire: Harper Adams University. Recuperado em Abril de 2020, de https://www.cabdirect.org/cabdirect/abstract/20193024435

\section{Submetido em: 18/Out/2019 \\ Aceito em: 19/Maio/2020}

Classificação JEL: R0; D60; D70. 